\title{
A Strategy for Power Generation Optimization in a Hybrid Wind- BESS Power Plant
}

\author{
Daniel Villanueva ${ }^{1 *}$, Andrés E. Feijóo ${ }^{1}$, and Neeraj D. Bokde ${ }^{2}$ \\ ${ }^{1}$ Electrical Engineering Department, University of Vigo, 36310-Vigo, Spain \\ ${ }^{2}$ National Institute of Technology, Nagpur, India
}

\begin{abstract}
The wind is an uncontrollable primary resource, although its energy can be stored. This fact can be used for the design of strategies for a better management of electric power networks. An option for achieving this goal is to install Battery Energy Storage Systems (BESS) in the wind farms (WF). When dealing with WFs combined with BESSs the most important is to manage the power production in order to meet the requirements of the network or those related with the owner of the plant. Both challenges constitute an optimization problem. This paper proposes an Evolutionary Algorithm (EA) to solve it, where a fitness function must be maximized under the consideration of certain constraints. The fitness function depends on the target of the power production, which may be either to help the network become more stable or to maximize the profit, assessing each scenario and accepting the best one. The constraints of the optimization problem are related to the levels of the BESSs: the maximum power transferred to or from it and the output power of the plant.
\end{abstract}

\section{Introduction}

Wind power generation presence in electrical power networks has been growing over the last decades, which has led their control to be more and more critical. Wind speed cannot be directly managed but it can be predicted with high accuracy, for which the efforts should be focused on network management, with the goal of injecting as much electric power from the wind as possible into it. But this action is not enough due to some reasons, such as the variability of the resource and its independence of the daily demand curve. In both cases, an amount of wind power can be wasted and/or used with a low efficiency, i.e., when it is not essential.

If the wind power production can be regulated or adapted to a specific daily demand curve, it can contribute to network stability and the profit can be higher. If it is the case, an interesting alternative of power injection strategy can be established. This consists of storing wind energy when there is a surplus, with the goal of injecting it into the power network when needed, according to the load demand curve. When not needed, it is preserved. This control can be considered as a contribution of the system to the steady-state stability of the network and can improve the profit of the power plant. If such a strategy is possible, then the consideration of WFs as very variable and uncontrollable power plants can be rethought.

Several means are generally used to store energy: different types of BESSs, capacitors, compressed air, liquid air, hydrogen, flywheels, hydroelectricity, superconductors, and others [1]. The intention of this paper is not to assess the most suitable mean to be used in combination with WFs, but several reasons support the use of BESSs:

i) The use of a BESS in combination with a WF arises as a solution applicable to all locations because there is no need of specific conditions in the installation.

ii) The efficiency of the system is very high.

iii) The number and/or size of the BESS can be adapted to the size of the WF, even though the bigger the $\mathrm{WF}$ is, the more efficient the installation is.

iv) They are especially valuable for small networks when other types of energy storage fail [2].

When dealing with a system formed by a WF and a BESS, the first issue to be considered is the battery size $[3,4]$, which is critical. However, it is not dealt with here and the value used in the case study is the existing one in a real power plant. The equations and constraints used in this paper appear in [4-13]. Another issue is the application of the BESS, that can be in an hourly, daily, weekly or monthly term [14], but the objective here is particularized for a daily term. Besides, prediction is not considered here, it is assumed that the wind speed is known with enough anticipation to apply the algorithm. This is realistic due to the daily term being analyzed.

Another issue to be considered is the model used for the BESS. Several models appear in the related literature $[9,15,16]$ and they have been simplified here, considering certain efficiency during the charging and discharging processes. Finally, it is also important to decide the optimal allocation of the BESS in the network 
and its configuration [17-19]. In this paper, the BESS is located adjacently to the WF and the chosen configuration is the one called dual inverter [19].

Then, to carry out a strategy based on the adaptation of the wind power produced by a power plant constituted by a WF and a BESS to the power requested along a period, is an optimization problem. The objective should be that the power plant generates more power when it is more valuable. However, the power plant will have some technical constraints that limit its operating capacity, most of them related with the BESS but also with the whole installation, turning the optimization problem into a non-linear one. Here, a specific EA is proposed, adapted to solve this problem. The reasons for using an EA are the constraints and the large amount of variables.

This paper is organized as follows: in section II, the concepts, expressions and constraints considered when dealing with a plant formed by a WF and a BESS are introduced; section III explains the application of the EA to the problem described in this paper; a specific case is shown in section IV and the conclusions are exposed in section $\mathrm{V}$.

\section{Power Supplied by a Wind Farm with a BESS}

As power plant consisting of a WF with a BESS is analyzed. The equations and constraints that model its behavior are presented in this section.

The power plant output is the sum of the power provided by the WF and by the BESS, as in (1).

$P_{g_{i}}=P_{b_{i}}+P_{w_{i}} \quad i=1, \ldots, n_{i}$

where $P_{g_{i}}$ is the power supplied by the power plant in the interval $i . P_{b_{i}}$, when positive, is the power provided by the BESS to the electrical network in the interval $i$. When it is negative, $\left|P_{b_{i}}\right|$ is the power provided by the WF to the BESS. Finally, $P_{w_{i}}$ is the power supplied by the WF in the interval $i$ and $n_{i}$ is the number of intervals.

The charging process of the BESS can be explained by (2). The energy in the BESS in a specific interval can be obtained as the sum of the energy stored in the previous interval and the power supplied to it along the interval, multiplied by the length of the interval and by a coefficient that accounts for the losses due to the efficiency of the process.

$E_{b_{i+1}}=E_{b_{i}}-\Delta T \cdot P_{b_{i}} \cdot \eta_{c} \quad i=1, \ldots, n_{i}$

where $E_{b_{i}}$ is the energy stored in the BESS in the interval $i, \eta_{c}$ is the efficiency of the charging process and $\Delta T$ is the interval length. Notice that $P_{b_{i}}$ is negative during the charging process. Therefore, $E_{b_{i+1}} \geq E_{b_{i}}$ always after applying (2).

If the BESS is discharging, the relationship among the variables is expressed in (3). In this case, the energy in the BESS in a specific interval can be obtained as the subtraction of the power provided by the BESS multiplied by the length of the interval, from the energy in the previous interval. A coefficient due to losses is also applied to the power provided by the BESS due to the efficiency of the process.

$E_{b_{i+1}}=E_{b_{i}}-\Delta T \cdot P_{b_{i}} / \eta_{d} \quad i=1, \ldots, n_{i}$

where $\eta_{d}$ is the efficiency of the discharging process. Therefore $P_{b_{i}}$ is the power provided by the BESS to the electrical network and $\Delta T \cdot P_{b_{i}} / \eta_{d}$ is the total amount of energy removed from the BESS. In this case, it is always $E_{b_{i+1}} \leq E_{b_{i}}$ after applying (3).

Besides, there are several constraints that must be taken into account to apply the given equations:

i) Constraints related to the level of the BESS (4): The BESS has a minimum level of charge in order to extend its useful life. It also has a maximum level of charge, i.e., its rated value.

$E_{b_{\min }} \leq E_{b_{i}} \leq E_{b_{\max }} \quad i=1, \ldots, n_{i}$

where $E_{b_{\text {min }}}$ is the minimum level of charge of the BESS and $E_{b_{\max }}$ its maximum one.

ii) Constraints related to the charging and discharging processes of the BESS (5): Due to the installation configuration and to the proper BESS, there are limits regarding the maximum power that can be transferred from the WF to the BESS and from it to the electrical network.

$P_{b_{\min }} \leq P_{b_{i}} \leq P_{b_{\max }} \quad i=1, \ldots, n_{i}$

where $P_{b_{\text {min }}}<0$ is the minimum value of the power provided by the BESS, i.e., the power limit to charge the BESS given in absolute value. $P_{b_{\max }}$ is the maximum value of the power provided by the BESS.

iii) Constraints related to the power supplied by the power plant (6): Again, due to the installation configuration, there are limits regarding the power that can be transferred from the power plant to the electrical network, i.e., the power plant cannot consume power and the output power cannot be higher than its rated power. Obviously, in this case, both limits are not critical, but out of that range, the installation has to be specifically designed to work in those situations, and that is not the classic configuration.

$0 \leq P_{g_{i}} \leq P_{g_{r}} \quad i=1, \ldots, n_{i}$

where $P_{g_{r}}$ is the rated power of the power plant.

\section{Application of the Evolutionary Algorithm}

An EA [20] includes processes related to physical or biological evolution such us crossover, mutation, 
recombination and the selection of the fittest individuals, to obtain the best solution of an optimization problem.

In the case studied in this paper, the application of the EA is very specific, due to the fact that a population of only one individual is needed, where a recombination of its elements is performed on each iteration. Then, the fitness of the new individual is assessed and it is accepted as the only member of the next generation when it is better than the parent's one. If the parent keeps fitting better than the child, the parent is the only member of the next generation. This algorithm does not correspond to a genetic algorithm itself but has some of its features.

More in depth, it can be said that the first individual is the vector formed by the power supplied by the WF in each of the intervals of the period, and, on each iteration, the vector is reorganized in order to obtain a better scenario, where the power supplied by the power plant is more suitable to the needs of the electrical network. In order to reorganize, part of the power provided by the WF during a specific interval is removed and added to another one. Normally, the period considered is one day, and the intervals last for ten minutes.

Therefore, on each iteration, a random value of power supplied by the WF is taken from a random interval and added to another random one. Then, the fitness function of the new vector formed by the $P_{g_{i}}$ elements is calculated. The fitness function is obtained as in (7).

$f=\sum_{i}\left(\omega_{i}^{2} \cdot P_{g_{i}}\right) \quad i=1, \ldots, n_{i}$

where $f$ is the fitness function and $\omega_{i}^{2}$ is the weight of the interval $i$. The weight of an interval is related to the importance of supplying power in that specific interval with respect to the rest. The weights can be obtained from the daily demand curve or from the prices of the power along the day, depending on whether the target is the contribution to the stability of the network or to obtain the maximum profit from the power plant, respectively. The reason why a squared value as a weight is included is that the highest values of power production in a specific interval are difficult to reach, so this speeds up the algorithm.

Finally, the fitness function is compared with its parent's one in order to decide which one is the only member of the new generation. The one with highest fitness function is retained and the other, rejected. The process is depicted in the scheme of Fig. 1 where ' $\mathrm{ff}$ ' stands for fitness function.

In Fig. 1 the intervals last for one hour in order to better appreciate the process. An initial situation (top graph) with a given fitness function (ff) can be seen. Then, a random value of power $(15 \mathrm{MW})$ is suggested to be moved from a random interval $(2-3 \mathrm{~h})$ to another random interval (15-16h) (middle graph), and the fitness function in this hypothetic situation is obtained ( $\mathrm{ff}^{\prime}$ ). Finally, two possible situations can be reached (bottom graphs): in case that $\mathrm{ff}^{\prime}>\mathrm{ff}$, the hypothetic situation is accepted (left one) and in other case, discarded (right one).
The process is repeated until either the fitness function does not increase its value in a given number of iterations or a maximum number of iterations is reached. For example, the evolution of the fitness function can be assessed every 100 iterations and the algorithm will have no more than 10 million iterations.

The result of the algorithm is the vector with the new values of power supplied by the power plant and, as a consequence, the exchange of power between the WF and the BESS. In fact, this EA represents the real behavior expected from the power plant itself due to the fact that the BESS is taking energy from the WF along a specific interval, and is giving back that energy along another interval. The algorithm decides, on each iteration, if a concrete scenario is more suitable than the previous one, considering the objective established. This situation, together with the tendency of the EAs to reach the best solution, provides a useful tool to solve the proposed problem.

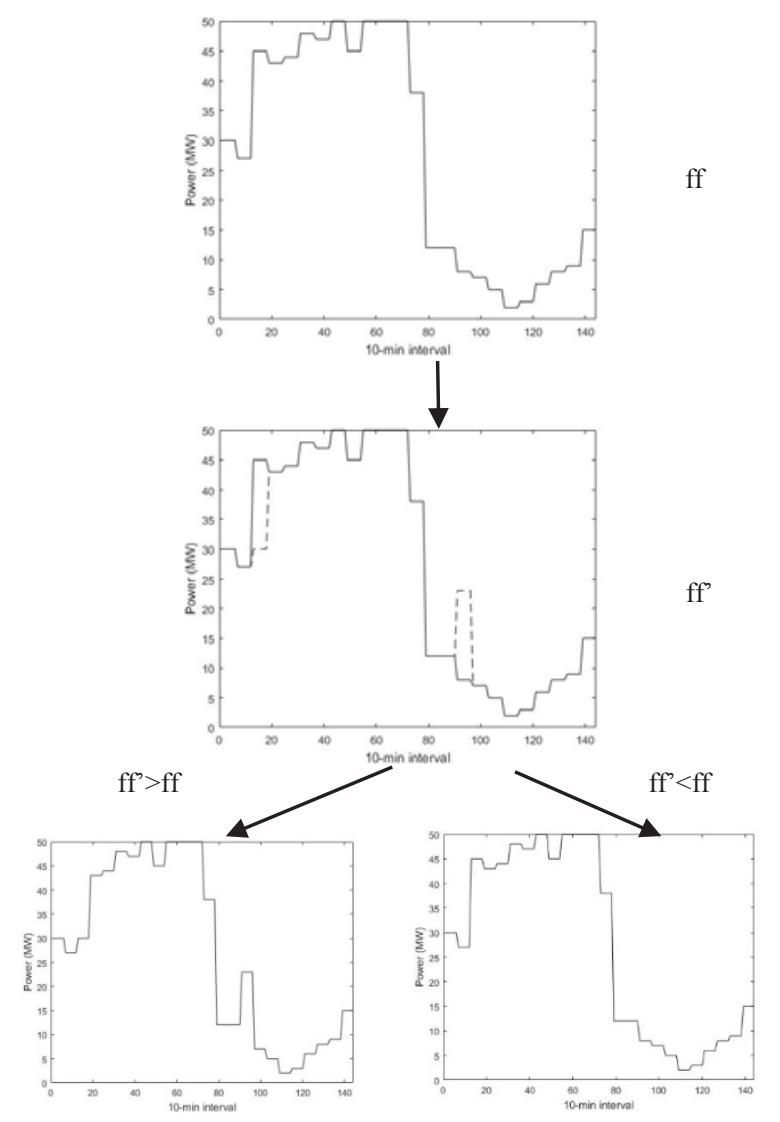

Fig. 1. Reorganization of the power supplied by the Power Plant performed on each iteration of the EA.

Moreover, as the algorithm is representing the desired behavior, the losses must be considered, due to the conversion of the energy from electric to chemical, when charging the BESS, and vice versa for the discharging process. In order to do that, the efficiencies of both processes $\left(\eta_{c}, \eta_{d}\right)$ are taken into account in the algorithm as shown in equations (2) and (3). 
Mathematically, the optimization problem is written as follows:

$$
\begin{gathered}
\max f=\sum_{i}\left(\omega_{i}^{2} \cdot P_{g_{i}}\right) \\
\text { s.t. }\left\{\begin{array}{c}
E_{b_{\min }} \leq E_{b_{i}} \leq E_{b_{\max }} \\
P_{b_{\min }} \leq P_{b_{i}} \leq P_{b_{\max }} \\
0 \leq P_{g_{i}} \leq P_{g_{r}}
\end{array}\right.
\end{gathered}
$$

considering the relationships in eqs. (1), (2) and (3).

\section{Simulation Results}

In order to check the results provided by the algorithm, a case study is proposed in this section. A WF is considered, constituted by $n_{w t}=25 \mathrm{WTs}$, each one with $P_{r}=2000 \mathrm{~kW}, v_{c i}=4 \mathrm{~m} / \mathrm{s}, v_{r}=$ $15 \mathrm{~m} / \mathrm{s}$ and $v_{c o}=25 \mathrm{~m} / \mathrm{s}$. A location is considered, where the wind speed is described by a Weibull distribution of parameters $C=12 \mathrm{~m} / \mathrm{s}$ and $k=2$. In order to simplify the calculations without loss of accuracy, the wind speed value is equal in all WTs.

The wind speed data are simulated using an autoregressive model of order 1, AR(1) [21], as in equation (8).

$x_{i}=\varphi \cdot x_{i-1}+\varepsilon_{i} \quad i=1, \ldots, n_{i}$

where $x$ is a Normal variable, $x_{i}$ is its value in the interval i, $\varphi$ is the autoregressive coefficient or lag-one autocorrelation, and $\varepsilon_{i}$ is a white noise signal in the interval $i$. The autoregressive coefficient used is $\varphi=0.95$ and the white noise is simulated by using random Normal data with parameters $\mu=0$ and $\sigma=\sqrt{1-\varphi^{2}}$. And converting the Normal data into Weibull data through a change of variables [22] as in (9).

$u_{i}=C\left(-\log \left(\left(1-\operatorname{erf}\left(\frac{x_{i}-\mu}{\sqrt{2} \sigma}\right)\right) / 2\right)\right)^{1 / k}$

where $\log ()$ is the natural logarithm and $\operatorname{erf}()$ is the error function defined by (10).

$\operatorname{erf}(x)=\frac{2}{\sqrt{\pi}} \int_{0}^{x} \exp \left(-t^{2}\right) d t$

where $\exp ()$ is the exponential function.

To obtain the power supplied by each WT the quadratic model for power curves is applied [23], according to (11).

$P_{w_{i j}}=\left\{\begin{array}{cc}0 & u_{i} \leq u_{c i} \\ P_{r} \cdot \frac{u_{i}^{2}-u_{c i}^{2}}{u_{r}^{2}-u_{c i}^{2}} & u_{c i} \leq u_{i} \leq u_{r} \\ P_{r} & u_{r} \leq u_{i} \leq u_{c o} \\ 0 & u_{c o} \leq u_{i}\end{array}\right.$ where $P_{w_{i j}}$ is the wind power supplied in the interval $i$ by the WT $j$, which corresponds to the wind speed $u_{i}$. Besides, as the wind speed value is considered equal in all the WTs simultaneously, the power supplied by the WF in the interval $i$ is $P_{w_{i}}=\sum_{j} P_{w_{i j}}=n_{w t} \cdot P_{w_{i j}}$.

The parameters of the BESS are: the minimum level, $E_{b_{\min }}=30 \mathrm{MWh}$, the maximum level, $E_{b_{\max }}=$ $150 M W h$, the charging limit of the BESS, $P_{b_{\min }}=$ $-25 M W$, and its discharging limit, $P_{b_{\max }}=25 \mathrm{MW}$.

The parameters of the power plant are: the rated power, $P_{g_{r}}=n_{w t} \cdot P_{r}=50 \mathrm{MW}$, the efficiency of the charging process, $\eta_{c}=0.90$ and the efficiency of the discharging process, $\eta_{d}=0.90$.

In this case, the vector of weights is defined with the objective of contributing to the stability of the network. Then, using a daily demand curve [24], it can be established that the minimum value of demand corresponds to $\omega_{i}=0$ and the maximum value of demand to $\omega_{i}=10$. The rest of weights are defined linearly between these two values. Notice the fact that the fitness function includes the squared weights, so the real weights used are those shown in Fig. 2, where the difference between the weights, $\omega_{i}^{2}$, and the coefficients, $\omega_{i}$ is depicted. As it has been said, this is done in order to speed up the algorithm: the highest values of power production are delayed to be reached using $\omega_{i}$ instead of $\omega_{i}^{2}$

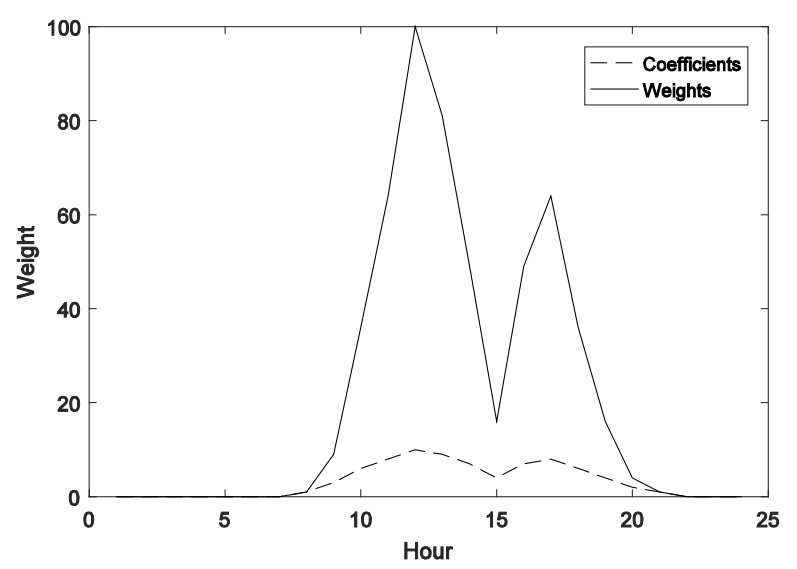

Fig. 2. Coefficients and weights as a function of the hour.

The following additional variables are needed: the total period considered is one day, each interval lasts for ten minutes, the evolution of the fitness function is assessed every 100 iterations, the maximum number of iterations is ten million and the initial state considered for the BESS corresponds to its minimum charge.

Then, once the input data is defined, the algorithm can be applied. In Figs. 3, 4 and 5 there are three applications of the algorithm to different random input data. 


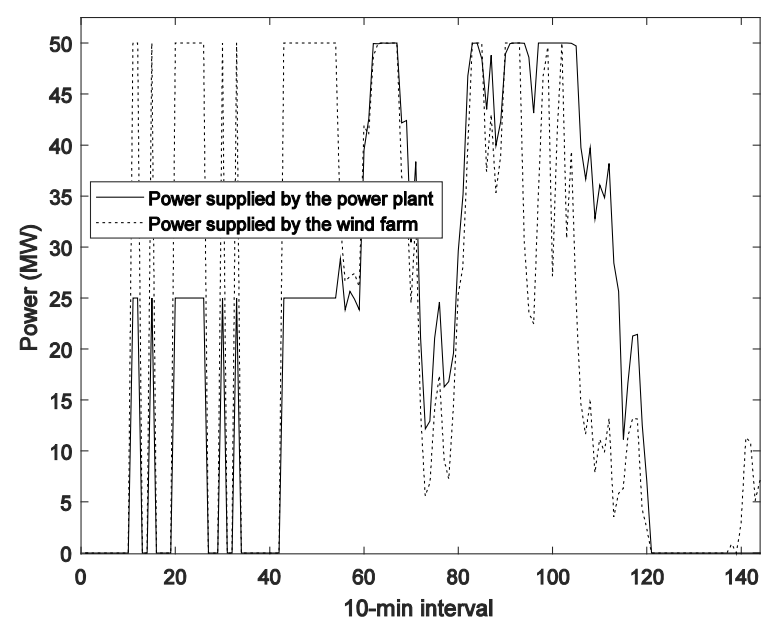

Fig. 3. EA applied to case 1.

In the first case, it can be seen a vector of power supplied by the WF where the rated power is generated during the night ( 0 to 50, 10-min interval) and also some power is produced during the hours with higher demand (50 to 12010 -min interval). After applying the algorithm, some of the power supplied by the WF during the night is moved to the latter intervals, increasing the production of the power plant in those ones. However, there are some limits to this reassignment: the limit of $25 \mathrm{MW}$ charging the BESS is reached repeatedly during the night, therefore, not all the power supplied by the WF can be used to charge the BESS. Besides, the limit in the power supplied by the power plant $(50 \mathrm{MW})$ is also reached several times during the periods with high demand. Obviously, there are some other cases where there is not a limit reached.

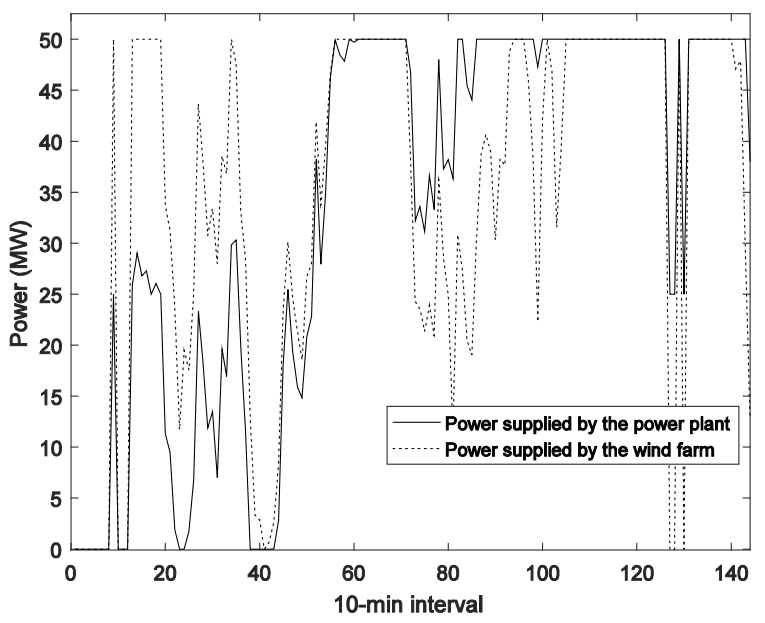

Fig. 4. EA applied to case 2.

In the second case, the scenario is a bit different. There is a large amount of power produced by the WF during the key intervals and a small one generated during the rest. Notice that the total production of energy may be different from one case to another. Here, after applying the algorithm, part of the power produced in the less interesting intervals is moved to the other ones, supplementing them when the rated power of the power plant is not reached. In Fig. 4, it can be seen as some kind of filling of a surface. Although at a first sight it may not be evident, some limits are also reached here, such as the charging limit of the BESS, in the interval 8, discharging, in the interval 100, rated power, in the interval 104 and output power higher than zero, in the interval 0 .

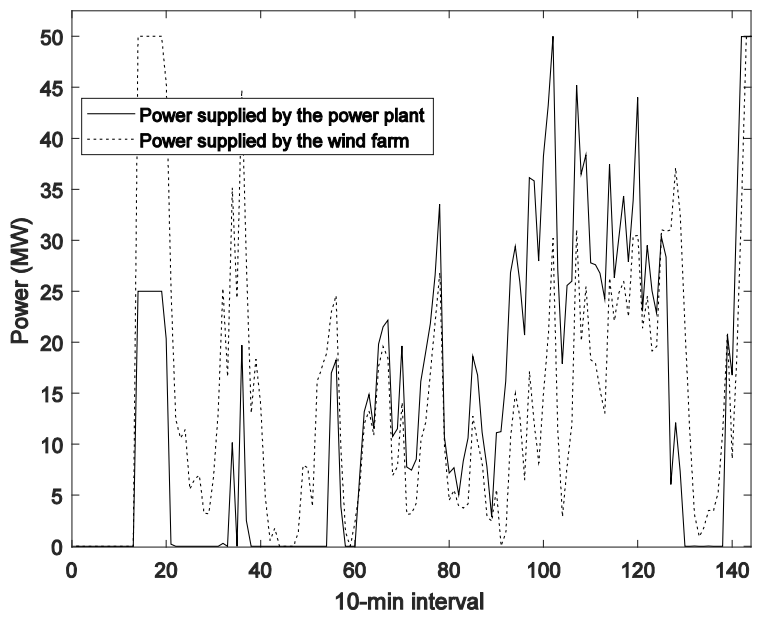

Fig. 5. EA applied to case 3.

In the last case the total energy supplied by the WF is lower than in the second case. In fact, it almost never reaches the rated power. Here, the power produced during the night is used to charge the BESS and it gives it back during the period of high demand. Notice the fact that, in this case, the limits avoid the possibility of no generation during all the night. Besides, the small amount of energy available in the BESS, together with the discharging limits provokes that the rated power is almost never reached. However, the power supplied by the power plant makes tentative attempts to adapt its shape to the daily demand curve.

Moreover, in order to assess the performance of the algorithm, its efficiency is defined by eq. (12).

$\rho_{i}=\frac{\text { Energy supplied by the power plant along day } i}{\text { Energy supplied by the WF along day } i}$

Efficiencies in the three cases are $\rho_{1}=0.9660$, $\rho_{2}=0.9809$ and $\rho_{3}=0.9377$. Even though the efficiencies when charging and discharging the BESS are lower, not all the power is supplied through the BESS, most of it is provided directly to the electrical network and that is the reason of the higher performance of the power plant. Besides, in Fig. 6, it can be seen the evolution of the fitness function in the three cases. 


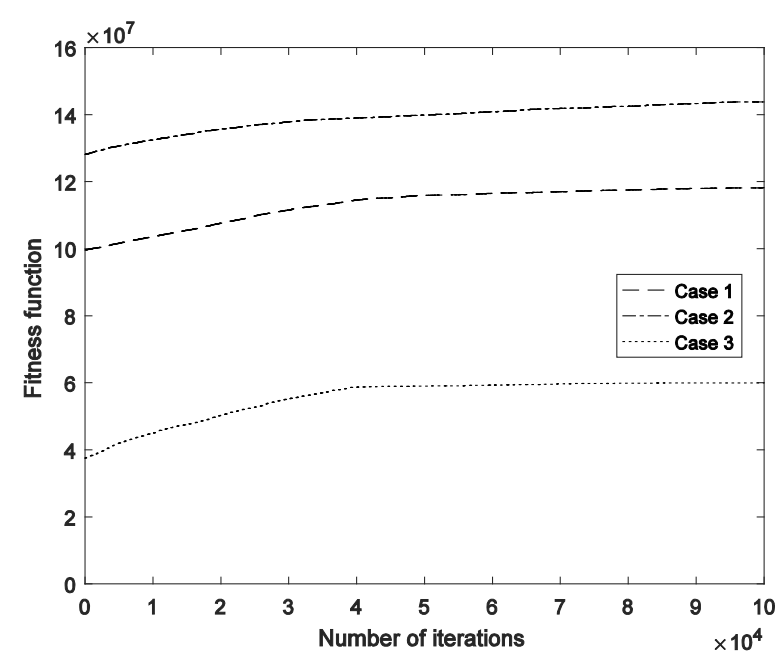

Fig. 6. Evolution of the fitness function in cases 1, 2 and 3 along the algorithm.

In all cases the fitness function behaves in the same manner: first, it increases very fast along the number of iterations 1 to 100 due to evident 'movements of power', then, it enters into a stage of slow increase (from 100 to 100,000 iteration) and, finally, it tends to a steady value (around 100,000 iteration to the end of the algorithm). In Fig. 6 the intermediate stage can be easily seen.

In the three cases, the solution proposed improves the contribution of the power plant to the stability of the network because more power is supplied when the demand is higher. And, obviously, the value of the fitness function is higher than just considering the power provided by the WF.

\section{Conclusions}

The combined use of WFs and BESS, in order to adjust generation and demand of power, seems to be of much interest, as there is no possibilty of establishing any control over the wind power.

In this paper, an EA is proposed to manage the production of a power plant constituted by a WF equipped with a BESS. The final result is obtained by optimizing a fitness function that prioritizes some periods of generation. The fitness function can be different depending on the main objective, i.e., the stability of the network or the maximum profit. Besides, the following constraints are considered in the algorithm: minimum and maximum levels of energy in the BESS, maximum power transferred when charging or discharging the BESS and minimum and maximum power supplied by the power plant.

If this kind of power plant works in line with the results of the proposed algorithm, the stability of the electrical network will be clearly reinforced and/or the maximum profit will be obtained by the owner. Additionally, in most cases both statements will be achieved simultaneously.

\section{References}

1. Banham-Hall, D.D., Taylor, G.A., Smith, C.A., Irving, M.R., Flow BESS for enhancing wind power integration (2012) IEEE Transactions on Power Systems, 27 (3), art. no. 6158621, pp. 1690-1697.

2. Mohod, S.W., Aware, M.V., Micro wind power generator with battery energy storage for critical load, (2012) IEEE Systems Journal, 6 (1), art. no. 6003747, pp. 118-125.

3. Protogeropoulos, C., Brinkworth, B.J., Marshall, R.H., Sizing and techno-economical optimization for hybrid solar photovoltaic/wind power systems with battery storage, (1997) International Journal of Energy Research, 21 (6), pp. 465-479.

4. Yao, D.L., Choi, S.S., Tseng, K.J., Lie, T.T., A statistical approach to the design of a dispatchable wind power-battery energy storage system, (2009) IEEE Transactions on Energy Conversion, 24 (4), art. no. 5336289, pp. 916-925.

5. Prasad, A.R., Natarajan, E., Optimization of integrated photovoltaic-wind power generation systems with battery storage (2006) Energy, 31 (12), pp. 1607-1618.

6. Khalid, M., Savkin, A.V., A model predictive control approach to the problem of wind power smoothing with controlled battery storage (2010) Renewable Energy, 35 (7), pp. 1520-1526.

7. Nguyen, M.Y., Nguyen, D.H., Yoon, Y.T., A new battery energy storage charging/discharging scheme for wind power producers in real-time markets (2012) Energies, 5 (12), pp. 5439-5452.

8. Jiang, Q., Wang, H., Two-time-scale coordination control for a battery energy storage system to mitigate wind power fluctuations (2013) IEEE Transactions on Energy Conversion, 28 (1), art. no. 6365250, pp. 52-61.

9. Alimisis, V., Hatziargyriou, N.D., Evaluation of a hybrid power plant comprising used EV-BESS to complement wind power (2013) IEEE Transactions on Sustainable Energy, 4 (2), art. no. 6331027, pp. 286-293.

10. Li, X., Hui, D., Lai, X., Battery energy storage station (BESS)-based smoothing control of photovoltaic (PV) and wind power generation fluctuations (2013) IEEE Transactions on Sustainable Energy, 4 (2), art. no. 6473871, pp. 464473.

11. Wee, K.W., Choi, S.S., Vilathgamuwa, D.M., Design of a least-cost battery-supercapacitor energy storage system for realizing dispatchable wind power (2013) IEEE Transactions on Sustainable Energy, 4 (3), art. no. 6487428, pp. 786-796.

12. Kou, P., Liang, D., Gao, F., Gao, L., Coordinated Predictive Control of DFIG-Based Wind-Battery Hybrid Systems: Using Non-Gaussian Wind Power Predictive Distributions (2015) IEEE Transactions on Energy Conversion, 30 (2), art. no. 7031952, pp. 681-695. 
13. Sun, Y., Zhong, J., Li, Z., Tian, W., Shahidehpour, M., Stochastic Scheduling of Battery-Based Energy Storage Transportation System with the Penetration of Wind Power (2017) IEEE Transactions on Sustainable Energy, 8 (1), art. no. 7501878, pp. 135144.

14. Zhou, W., Yang, H., Fang, Z., Battery behavior prediction and battery working states analysis of a hybrid solar-wind power generation system (2008) Renewable Energy, 33 (6), pp. 1413-1423.

15. Swierczynski, M., Stroe, D.I., Stan, A.-I., Teodorescu, R., Sauer, D.U., Selection and performance-degradation modeling of limo2/li 4ti5o12 and lifepo4/c battery cells as suitable energy storage systems for grid integration with wind power plants: An example for the primary frequency regulation service (2014) IEEE Transactions on Sustainable Energy, 5 (1), art. no. 6583344, pp. 90101.

16. Teleke, S., Baran, M.E., Bhattacharya, S., Huang, A.Q., Optimal control of battery energy storage for wind farm dispatching (2010) IEEE Transactions on Energy Conversion, 25 (3), art. no. 5432995, pp. 787-794.

17. Zhang, Y., Dong, Z.Y., Luo, F., Zheng, Y., Meng, K., Wong, K.P., Optimal allocation of battery energy storage systems in distribution networks with high wind power penetration (2016) IET Renewable Power Generation, 10 (8), pp. 1105-1113.
18. Nguyen, C.-L., Lee, H.-H., A Novel Dual-Battery Energy Storage System for Wind Power Applications (2016) IEEE Transactions on Industrial Electronics, 63 (10), art. no. 7473865, pp. 61366147.

19. Gamini Jayasinghe, S.D., Mahinda Vilathgamuwa, D., Madawala, U.K., Direct integration of battery energy storage systems in distributed power generation (2011) IEEE Transactions on Energy Conversion, 26 (2), art. no. 5742991, pp. 677-685.

20. Juste, K.A., An evolutionary programming solution to the unit commitment problem (1999) IEEE Transactions on Power Systems, 14 (4), pp. 14521459.

21. P. F. Correia, J. M. Ferreira, and de Jesús, "Simulation of correlated wind speed and power variates in wind parks,"Elect.PowerSyst.Res., vol. 80, no. 5, pp. 592-598, May 2010.

22. Villanueva, D., Feijóo, A., Pazos, J.L. Simulation of correlated wind speed data for economic dispatch evaluation (2012) IEEE Transactions on Sustainable Energy, 3 (1), art. no. 6102285, pp. 142-149.

23. Villanueva, D., Pazos, J.L., Feijóo, A., Probabilistic load flow including wind power generation (2011) IEEE Transactions on Power Systems, 26 (3), art. no. 5682076, pp. 1659-1667.

24. Lin, C.E., Hong, Y.Y., Chuko, C.C., Real-time economic dispatch - A fast base-case approach (1987) Electric Power Systems Research, 12 (3), pp. 167-173. 